ISSN: $1560-9111$

\title{
Patología oral en niños y adolescentes con VIH en tratamiento antirretroviral de gran actividad
}

\author{
Artículo Original
}

\author{
Oral pathology in children and adolescents with HIV on antiretroviral \\ therapy highly active
}

\begin{abstract}
Resumen
Objetivo: Determinar patologías orales en niños y adolescentes con virus de la inmunodeficiencia hu-mana (VIH) en tratamiento antirretroviral de gran actividad (TARGA) según su estado clínico e inmunológico y las características del TARGA que recibían. Materiales y método: Es un estudio descriptivo, transversal y observacional. Se evaluaron 20 pacientes pediátricos del Hospital Nacional Hipólito Unanue, de ambos sexos, entre 2 y 13 años. Las patologías se diagnosticaron mediante el sistema recomendado por el Instituto de Problemas Orales relacionados con la infección por el VIH de la Comunidad Económica Europea, el Centro de Colaboración en Manifestaciones Orales del VIH de la Organización Mundial de la Salud y la clasificación de manifestaciones orofaciales en niños con VIH de Ramos y colaboradores. Se elaboró una base de datos, usando estadística descriptiva. Resultados: La patología oral encontrada fue herpes simple (5\%), caries dental (100\%) y gingivitis (95 $\%)$. La dentición decidua estaba presente en el $20 \%$, con un índice ceo de $7 \pm 2$, los demás presentaban dentición mixta con índices CPOD y ceo de $5 \pm 6,12$ y 6,81 $\pm 5,58$ respectivamente. Respecto al Índice de Higiene Oral de Greene y Vermillion, el $85 \%$ tuvo nivel deficiente y $15 \%$ regular, siendo el valor promedio $2,52 \pm 0,46$. Conclusiones: Se atribuye la presencia mínima de patología oral asociada al VIH al TARGA. Se resalta que el tiempo y tipo de tratamiento son esenciales para la reconstitución del sistema inmunitario. Las lesiones cariosas y la gingivitis son posiblemente relacionadas a la deficiente higiene oral en la muestra estudiada.

Palabras clave: Caries dental, Herpes simple, Patología bucal, Terapia antirretroviral, VIH.
\end{abstract}

\begin{abstract}
Objective: Determine oral pathologies in children and teenagers with human immunodeficiency virus (HIV) infection in antiretroviral therapy (HAART) according to their clinical and immunological status and characteristics of the receiving HAART. Materials and method: A descriptive, transversal and obser-vational study. 20 pediatric patients Hipolito Unanue National Hospital of both sexes between 2 and 13 years were evaluated. Pathologies were diagnosed by recommended by the Clearinghouse on Oral Problems related to HIV infection in the European Community and The Collaborating Center on Oral Manifestation of the Human Immunodeficiency Virus from The World Health Organization and classification of orofacial manifestations in system children with HIV Ramos et al. (1999) a database will be developed, using descriptive statistics. Results: The pathology was found herpes simplex (5\%), dental caries (100\%) and gingivitis (95\%). The $20 \%$ of the whole population had Primary Dentition, with a "dmf index" of $7 \pm 2$. The other 80 $\%$ of the whole population had mixed dentition with "DMF" and "dmf" index of $5 \pm 6,12$ and 6,81 $\pm 5,58$ respectively. With regard to the OHI-S Greene and Ver-milion index $85 \%$ had a deficient level and $15 \%$ had a regular level, with an average value of 2,52 $\pm 0,46$. Conclusions: The minimal presence of HIV-associated oral pathology is attributed to HAART. It emphasized that the time and type of treatment are essential for the reconstitution of the immune system. Carious lesions and gingivitis are possibly related to poor oral hygiene in the sample studied.
\end{abstract}

Keywords: Dental Caries, Herpes Simplex, Oral Pathology, Antiretroviral Therapy, HIV.

\section{Introducción}

El impacto de la infección del VIH y sus consecuencias como ninguna otra enfermedad en la historia viene propagándose en el mundo. Según estimaciones del Programa Conjunto de las Naciones Unidas sobre el VIH/sida, ONUSIDA en su Reporte Global del ańo 2014 informa que a nivel mundial existían aproximadamente 2,6 millones $(2,4-2,8$ millones $)$ de nińos menores de 15 ańos infectados con VIH. En América latina existían 33000 (29 000 - 40 000) casos y en el Caribe 13 $000(11000-15000){ }^{1}$

El VIH se clasifica en VIH-1 y VIH-2. El VIH-1 es responsable de la pandemia, resaltando su variabilidad gené-
1. Escuela Académico Profesional de Odontología de la Universidad Nacional Mayor de San Marcos

2. Departamento de Ciencias Básicas de la Facultad de Odontología de la Universidad Nacional Mayor de San Marcos

\section{Correspondencia:}

George Paul Márquez Peña

Av. Germán Amézaga 375, Lima 1, Perú

Correo electrónico: gpaulmarquez@gmail.com

Coautor:

Marieta Petkova-Gueorguieva:

mpetkovag@unmsm.edu.pe

Fecha de recepción: 07/12/2015

Fecha de aceptación: 18/04/2016

tica que le confiere gran agresividad y resistencia. El VIH-2 es principalmente observado en África Occidental, menos patogénico y transmisible que el VIH1 , además presenta períodos de latencia clínica más largos, menor tasa de transmisión vertical y es resistente a los inhibidores de la transcriptasa reversa no nucleósidos (ITRNN). Ambos tipos de 
VIH presentan los mismos modos de transmisión y están asociados a similares infecciones oportunistas y desarrollo de sida. ${ }^{2,3}$

El VIH tiene mecanismos de transmisión que están bien definidos. La vía sexual es la forma más común, pero no habitual en los niños, la vía sanguínea a través de transfusión de sangre o productos derivados de ella, así como el uso de drogas intravenosas o accidentes laborales, esta vía es casi nula en la población pediátrica y la vía madre niño llamada transmisión vertical, la cual es el principal medio por el cual los nińos adquieren el VIH y puede ocurrir en tres momentos distintos: 1. Transmisión prenatal, intrauterina o transplacentaria, la infección puede ocurrir durante la gestación. 2. Transmisión in-traparto o perinatal, la infección puede producirse durante el parto.3. Trans-misión posnatal o posparto, el VIH se puede transmitir a través de la lactancia materna. ${ }^{4-7}$

La infección por el VIH en el niño tiene un curso rápidamente progresivo en relación con el adulto, presentando un corto período de incubación. Los nińos infectados por vía vertical tienen una forma bimodal de presentación y evolución, muy probablemente relacionada con el momento de la infección. Se observa una forma precoz de evolución rápidamente progresiva que afecta aproximadamente al 10-20\% de los niños infectados verticalmente, posiblemente adquiriendo el virus de forma in- trauterina, presentando en el momento del nacimiento signos virológicos e inmunológicos de infección y una forma tardía de evolución más lenta, que es la forma más frecuente de la infección perinatal, aproximadamente $80 \%$ y al nacimiento no presentan signos clínicos, inmunológicos ni virológicos de infección. ${ }^{8}$

La CDC (Centers for Disease Control and Prevention) de Atlanta en 1994 clasifica a la infección pediátrica por el virus del VIH en categorías clínicas: N (asintomático), A (sintomatología leve), B (sintomatología moderada) y C (sintomatología severa). También la clasificó en categorías inmunológicas: 1 (sin supresión), 2 (supresión moderada) y 3 (supresión severa). ${ }^{8}$

Con la aparición de los fármacos antirretrovirales (drogas que actúan a varios niveles de la dinámica de la acción viral con la célula huésped) se ha reducido notablemente la mortalidad de los niños infectados por el VIH. Estos medicamentos utilizados en la población pediátrica se clasifican en: inhibidores de la transcriptasa reversa nucleósidos (ITRN), inhibidores de la transcriptasa reversa no nucleósidos (ITRNN), inhibidores de la proteasa (IP) e inhibidores de la unión y de la fusión. La combinación de ellos fue denominado terapia antirretroviral de gran actividad (TARGA) cuyos objetivos son: Reducir la morbilidad y mortalidad asociada a VIH/SIDA, mantener una supresión viral máxima y prolongada (carga viral indetectable idealmente), restaurar y preservar el sistema inmune (incremento de CD4 idealmente sin inmunosupresión), promover o restaurar el normal crecimiento y desarrollo, minimizar la toxicidad asociada a medicamentos, prolongar la sobrevida $\mathrm{y}$ mejorar la calidad de vida. ${ }^{9}$

En 1993 el Instituto de Problemas Orales relacionados con la infección por el VIH de la Comunidad Económica Europea (CEE) y el Centro de Colaboración en Manifestaciones Orales del VIH de la Organización Mundial de la Salud (OMS) realizaron la clasificación de las patologías orales asociadas a la infección por el VIH en niños. A esta clasificación añadiremos la caries dental, debido a su alta prevalencia en nuestro ámbito y por estar muy frecuente en nuestros pequeños pacientes (Tabla 1). ${ }^{10-13}$

Los investigadores Gaitán et al. ${ }^{14}$, Bosco et al. ${ }^{15}$, Okunseri et al. ${ }^{16}$, Pongsiriwet et al. ${ }^{17}$ y Miziara et al. ${ }^{18}$ concluyen que el uso de TARGA produce una gran disminución de las patologías orales asociadas al VIH. Mientras que Ferreira et al. ${ }^{19}$ y Benito et al. ${ }^{20}$ reportan una ligera reducción de estas patologías. No obstante, Flanagan et al. ${ }^{21}$, Khongkunthian et al..$^{22}$, Chen et al. ${ }^{23}$ y Hanza et al..$^{24}$ obtienen como resultado en sus investigaciones que el uso de TARGA no produce disminución de estas lesiones orales. Sin embargo, Chen et al. ${ }^{23}$ manifiesta una disminución de lesiones cariosas.

Tabla 1. Clasificación de las patologías orales asociadas a la infección por VIH en niños.

\begin{tabular}{|c|c|c|}
\hline $\begin{array}{l}\text { Grupo 1: Patologías comúnmente asociadas } \\
\text { a la infección pediátrica por el VIH }\end{array}$ & $\begin{array}{l}\text { Grupo 2: Patologías menos comúnmente } \\
\text { asociadas a la infección pediátrica por el VIH }\end{array}$ & $\begin{array}{l}\text { Grupo 3: Patologías fuertemente asociadas } \\
\text { con la infección por el VIH pero rara en niños }\end{array}$ \\
\hline $\begin{array}{l}\text { - Candidiasis oral } \\
\text { - Pseudomembranosa } \\
\text { - Eritematosa } \\
\text { - Virus del herpes simple } \\
\text { - Primaria } \\
\text { - Secundaria } \\
\text { - Eritema gingival lineal } \\
\text { - Agrandamiento de la parótida } \\
\text { - Ulceraciones aftosas recurrentes } \\
\text { - Menor } \\
\text { - Mayor } \\
\text { - Herpetiforme }\end{array}$ & $\begin{array}{l}\text { - Infecciones bacterianas de tejidos orales } \\
\text { - Enfermedades periodontales } \\
\text { - Gingivitis ulcerativa necrotizante } \\
\text { - Periodontitis ulcerativa necrotizante } \\
\text { - Estomatitis necrosante } \\
\text { - Infecciones virales } \\
\text { - Citomegalovirus } \\
\text { - Virus del papiloma humano } \\
\text { - Molusco contagioso } \\
\text { - Virus varicela zóster } \\
\text { - Herpes zóster } \\
\text { - Varicela zóster } \\
\text { - Dermatitis seborreica } \\
\text { - Xerostomía }\end{array}$ & $\begin{array}{l}\text { - Neoplasias } \\
\text { - Linfoma no Hodgkin } \\
\text { - Sarcoma de Kaposi } \\
\text { - Leucoplasia pilosa } \\
\text { - Úlceras relacionadas con la tuberculosis }\end{array}$ \\
\hline
\end{tabular}


Es de gran importancia destacar algunas patologías orales como: 1. Candidiasis oral, la cual es la lesión más común en niños con sida y frecuentemente la primera manifestación de infección por VIH. Tiene tres presentaciones clínicas: candidiasis pseudomembranosa, eritematosa y queilitis angular, además está asociada a una progresión más rápida de inmunosupresión. 2. Virus del herpes simple. Es la infección viral aguda más frecuente de la mucosa bucal. Tiene dos formas de presentación: gingivoestomatitis herpética primaria y la recurrente o secundaria, no está relacionado al nivel de progresión del VIH. 3. Eritema gingival lineal, que puede estar acompañado de neutropenia y también se puede sobreinfectar con candidiasis. 4. Agrandamiento de la parótida. Es un indicador de la progresión más lenta del VIH. 5. Las úlceras aftosas recurrentes, que se manifiestan en tres formas clínicas: úlceras aftosas recurrentes menor, mayor y herpetiforme, las cuales pueden ser un signo clínico inicial de anemia o neutropenia en el nińo infectado por el VIH. 6. Citomegalovirus. Es un marcador de inmunosupresión severa. La presencia de lesiones orales está considerada como una señal de la enfermedad ya diseminada. 7. La xerostomía. El paciente presenta un mayor riesgo de desarrollar caries, gingivitis, candidiasis, labios rajados y disfagia. 8 . Linfoma no Hodgkin. Es la neoplasia más común en niños infectados por el $\mathrm{VIH}$, diferente a los adultos, no siempre es asociada con inmunosupresión severa. 9. Leucoplasia pilosa, considerada como un marcador de inmunosupresión grave. ${ }^{10,25}$

El objetivo de este estudio fue determinar las patologías orales en nińos y adolescentes con VIH que están en tratamiento antirretroviral de gran actividad, según el estado clínico e inmunológico en el que se encuentran y las características del TARGA que reciben en el Hospital Nacional Hipólito Unanue.

\section{Material y método}

Se realizó un estudio de tipo descriptivo y transversal. Se abarcó toda la población de estudio, conformada por 20 niños con VIH, 8 de sexo masculino y 12 de sexo femenino, cuyas edades estuvieron comprendidas entre los 2 y los 13 años, que se encontraban en TARGA en el Hospital Nacional Hipólito Unanue en el período 2008.
En los criterios de inclusión se tomó en cuenta:

1. Pacientes con historias clínicas registradas y codificadas.

2. Pacientes niños de ambos sexos. Todos positivos para anticuerpos contra el VIH, detectados con la prueba del PRC, ELISA y confirmado con el Western Blot (WB).

3. Pacientes que iniciaron terapia antirretroviral dentro del Hospital Nacional Hipólito Unanue y acudían a consulta de manera regular al servicio de pediatría.

4. Pacientes que tuvieron previo al inicio de la terapia un examen de recuento de $\mathrm{CD} 4$, y dosaje de carga viral.

Este estudio fue aprobado por el Comité de ética médica.

Previamente a la evaluación, los pacientes y sus padres y/o tutores fueron informados sobre el proyecto de investigación. Ambos firmaron una hoja de asentimiento y consentimiento informado respectivamente. Se elaboró una ficha odontológica, la cual fue calibrada y validada con un juicio de expertos. La ficha constaba de dos secciones. La primera parte contenía los datos de filiación, esquemas antirretrovirales, categorías clínicas e inmunológicas, conteo de CD4 y de carga viral, así como su seguimiento y evolución. La segunda parte constaba del examen clínico, el cual se dividía en examen extraoral e intraoral, índice de higiene oral de Greene y Vermillion, odontograma recomendado por el MINSA y diagnóstico. Se recopilaron datos necesarios de las historias clínicas del hospital de los pacientes, incluyendo esquemas TARGA de inicio y actuales, el conteo de CD4 y carga viral. Luego de ubicar al paciente en el sillón dental, se procedió al examen clínico. Las patologías orales y periorales se examinaron y diagnosticaron mediante el sistema recomendado por el Instituto de Problemas Orales relacionados con la infección por el VIH de la Comunidad Económica Europea (CEE) y el Centro de Colaboración en Manifestaciones Orales del VIH de la Organización Mundial de la Salud (OMS) y apoyados por la clasificación y diagnóstico de manifestaciones orofaciales en nińos infectados por el VIH propuesto por Ramos et al. El examen extrabucal consistió en una inspección visual de la cara del paciente y las regiones de la cabeza. Se realizó la palpa- ción de los ganglios linfáticos cervicales, submandibulares, submentonianos y la glándula parótida. El examen intraoral fue realizado utilizando un equipo de diagnóstico estéril que contenía espejos bucales planos $n^{\circ} 4$ y 5 , pinzas, exploradores bucales biactivos y bajalenguas. Los tejidos blandos fueron examinados en la secuencia siguiente: mucosa y surcos labiales (superior e inferior), parte labial de las comisuras y la mucosa bucal derecha e izquierda, lengua (superficie dorsal y ventral, bordes), suelo de la boca, paladar duro y blando, bordes alveolares y encías (superiores e inferiores). Las piezas dentarias fueron examinadas y los resultados se registraron en el odontograma recomendado por el MINSA. Cuando fue necesario se tomaron radiografías periapicales y de aleta mordible. Se establecieron índices de caries dental para la dentición temporal y permanente utilizando los índices CPOD y ceo, de acuerdo a los criterios de la Organización Mundial de la Salud (OMS). Se determinó el estado de higiene oral de los pacientes pediátricos empleando el índice de higiene oral simplificado de Greene y Vermillion (IHO-S). Se confeccionó una base de datos, organizándolos en tablas y gráficas, usando estadística descriptiva, hallando la media y desviación estándar $\mathrm{y}$ frecuencias y porcentajes.

\section{Resultados}

Del total de la población de 20 pacientes, el mayor porcentaje se encontraba en la categoría clínica B (sintomatología moderada), con un $55 \%$ (11) y en la categoría clínica inmunológica B-3 con un $25 \%$ (5) del total, mientras que la menor cantidad se halló en la categoría clínica A (sintomatología leve) con un $15 \%$ (3) y en las categorías clínicas inmunológicas $\mathrm{C}-1$ y $\mathrm{A}-3$ con un $5 \%$ (1) cada una. (Fig. 1)

El $100 \%$ (20) de los participantes presentaban lesiones cariosas. Se observó un mayor porcentaje de caries dental $25 \%$ (5) en la categoría clínica inmunológica B-3, lo cual podría ser porque la mayor cantidad de pacientes se ubica en esta categoría. Además, el $5 \%$ (1) presentaba herpes simple encontrándose en la categoría clínica inmunológica C-3. (Fig. 1)

La totalidad de los pacientes pediátricos con VIH presentaron caries dental independientemente del esquema TARGA que recibían, encontrándose la mayoría con el esquema $\mathrm{AZT}+3 \mathrm{TC}+\mathrm{NFV}$ $(55 \%)$ y la menor cantidad con el es- 
quema ddI+3TC+kaletra $(5 \%)$. Solo se presentó un caso de herpes simple (5\%) en un paciente al que se le administra-ba el esquema ddI+3TC+kaletra. (Fig. 2)

La mayoría de los niños experimentaron inflamación gingival moderada (95 $\%)$ y una higiene oral deficiente, con una acumulación de placa bacteriana en casi la totalidad de los pacientes. Según los valores del IHO-S (0-0,5 buena higiene, $0,6-1,5$ regular higiene $y$ 1,6-3,0 mala higiene) podemos men- cionar que $85 \%(17 / 20)$ presentaron una condición mala y $15 \%(3 / 20)$ una condición regular. El IHO-S promedio correspondió a 2,52 $\pm 0,458$.

La caries dental afectaba a la totalidad de nuestros pacientes pediátricos, los cuales fueron divididos en dos grupos: pacientes con dentición mixta 16/20 (80\%) y con dentición decidua 4/20 $(20 \%)$. El primer grupo presentó un índice CPOD de $5 \pm 6.12$, donde el segmento perteneciente a caries corres- pondió a 4,81 $\pm 5,93$, perdidos $0,18 \pm$ 0,75 y el segmento obturado 0 (Tabla 2). Este mismo grupo mostró un índice ceo de $6,81 \pm 5,58$, con un segmento cariado de 5,25 $\pm 4,71$, con extracción indicada $1,43 \pm 2,18$ y un segmento obturado de 0,12 \pm 0.5 (Tabla 2). Los pacientes con solo dentición decidua tuvieron un índice ceo de $7 \pm 2$, con un segmento perteneciente a caries de $5,5 \pm 3$, extracción indicada $1,5 \pm 3 \mathrm{y}$ dientes obturados 0 (Tabla 2).

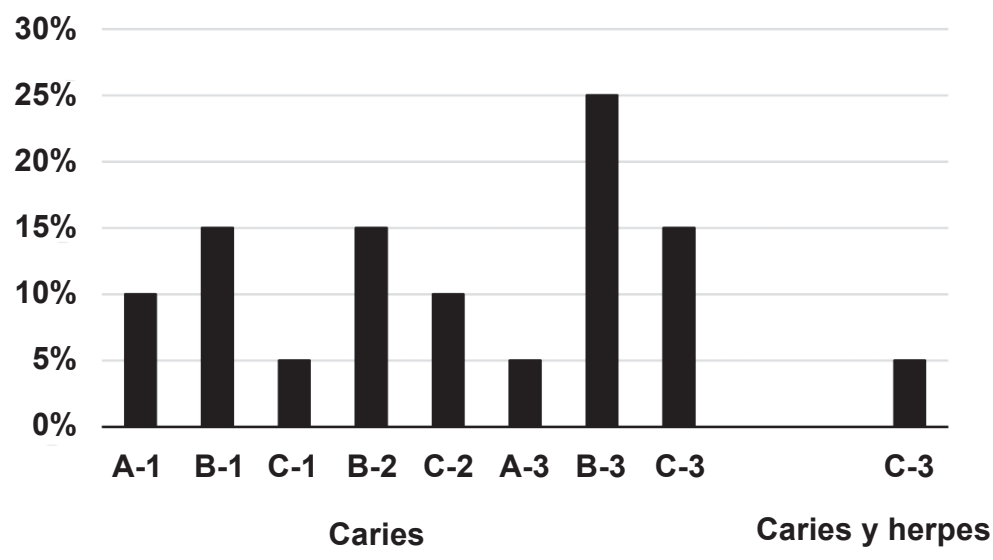

Fig. 1. Patologías orales encontradas, según categoría clínica e inmunológica.

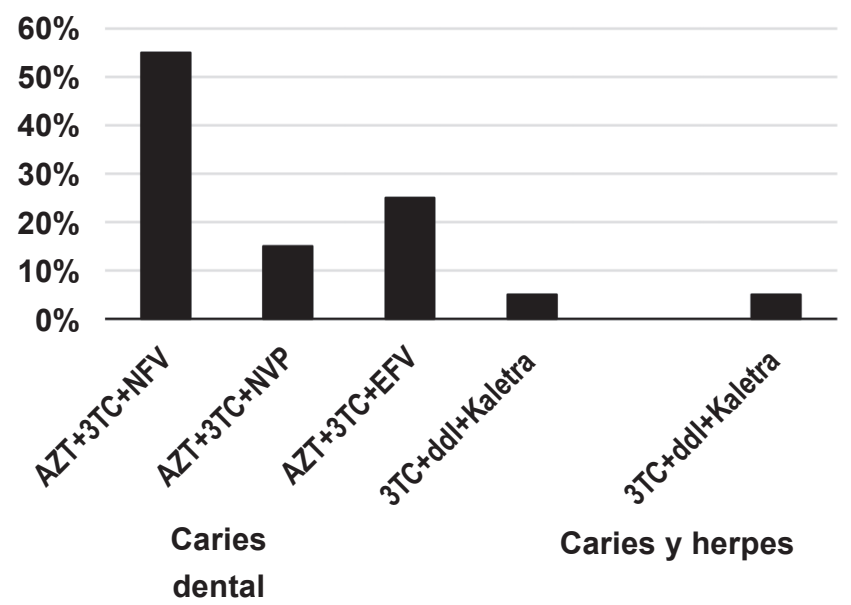

Fig. 2. Patologías orales encontradas, según tipo de esquema TARGA.

Tabla 2. Promedio de dientes CPOD y ceo en pacientes pediátricos con VIH que recibían TARGA con dentición mixta y decidua.

\begin{tabular}{|c|c|c|c|c|c|c|}
\hline \multirow{4}{*}{16 niños con dentición mixta } & C & $\mathbf{P}$ & 0 & Valor mínimo & Valor máximo & CPOD \\
\hline & $\begin{array}{l}4,81 \pm \\
5,93\end{array}$ & $\begin{array}{c}0,18 \pm \\
0,75\end{array}$ & 0 & 2 & 20 & $\begin{array}{l}5 \pm \\
6,12\end{array}$ \\
\hline & C & $E$ & 0 & Valor mínimo & Valor máximo & ceo \\
\hline & $\begin{array}{l}5,25 \pm \\
4.71\end{array}$ & $\begin{array}{l}1,43 \pm \\
2.18\end{array}$ & $0,12 \pm 0,5$ & 1 & 18 & $\begin{array}{l}6,81 \pm \\
5,58\end{array}$ \\
\hline \multirow{2}{*}{4 niños con dentición decidua } & C & $E$ & 0 & Valor mínimo & Valor máximo & ceo \\
\hline & $5,5 \pm 3$ & $1,5 \pm 3$ & 0 & 4 & 8 & $7 \pm 2$ \\
\hline
\end{tabular}




\section{Discusión}

En el presente trabajo se reportó la presencia mínima de patologías orales asociadas al VIH pediátrico en nuestra población, mostrándose un solo caso (1/20) de herpes simple $(5 \%$ de la población), lo cual coincide con los hallazgos de Gaitán et al. ${ }^{14}$ quienes manifestaron la identificación de solo 2 casos (2/16) de herpes simple en sus pacientes (prevalencia de 12,5\%). Igualmente, Benito et al. ${ }^{20}$ mencionaron la presencia de un solo caso (1/32) de herpes labial (4,76\%), similarmente Flanagan et $\mathrm{al}^{21}$ informaron que un sujeto $(1 / 38)$ presentó herpes gingival $(3 \%)$ y otro $(1 / 38)$ herpes labial (3\%). Mientras que Chen et al. ${ }^{23}$ identificaron 8 casos $(8 / 56)$ de herpes labial (14\%), y Miziara et al. ${ }^{18}$ reportaron 12 casos $(12 / 144)$ de herpes simple (8.3 \%), además, 9 casos (9/144) de gingivoestomatitis herpética $(6,25 \%)$ en niños.

En la investigación casi no se encontraron lesiones orales asociadas al VIH y esto puede ser atribuido al tratamiento antirretroviral de gran actividad, el cual contribuye a restaurar y preservar el sistema inmune (incremento de CD4, idealmente sin inmunosupresión) y mantener una supresión viral máxima y prolongada (carga viral indetectable idealmente).

Al comparar los índices CPOD y ceo con los valores propuestos por la OMS (0 $-1,1$ muy bajo, 1,2-2,6 bajo, 2,7-4,4 moderado, 4,5-6,5 alto y 6,6 - o más es muy alto) obtuvimos un valor alto $y$ muy alto. Estos índices reflejan una elevada prevalencia de lesiones cariosas en la población estudiada. $\mathrm{La}$ prevalencia de caries dental (100 \%) observada en este estudio puede ser asociada con la deficiente higiene o ral en la muestra. Los niños infectados con VIH están expuestos a diversos factores relacionados a una alta prevalencia de caries dental. ${ }^{17}$

El índice CPOD encontrado coincide con los hallazgos de Chen et $\mathrm{al}^{23}$, quienes reportaron un valor CPOD de 5,3, sin embargo, informaron un índice ceo de 2,8, con una prevalencia del $32 \%(18 / 56)$. Es importante señalar que Chen et al. ${ }^{23}$ manifestaron una disminución de lesiones cariosas en los pacientes pediátricos infectados con VIH en tratamiento antirretroviral. Esto puede ser, según Chen et al. ${ }^{23}$ por el mejor acceso a la atención médica que tuvieron estos niños. Okunseri et al. ${ }^{16}$ también reportó una prevalencia baja de caries dental de 23,5\% (24/102) y sugirió que podría deberse al acceso a agua fluorada u otras medidas cautelares a disposición de los niños.
Con respecto a la inflamación gingival, observamos cambios locales o generalizados en el color, forma y/o volumen, correspondiendo este diagnóstico a una gingivitis, la cual se presenta de forma moderada en un $95 \%(19 / 20)$ de los pacientes. En este estudio, el factor relacionado con la elevada prevalencia de gingivitis fue la inadecuada higiene oral ya que todos los pacientes recibían tratamiento antirretroviral de gran actividad. El hallazgo supera lo reportado por Flanagan et al., ${ }^{21}$ con una prevalencia alta de gingivitis en un $50 \%$ (19/38) de su población, Chen et al. ${ }^{23}$ informaron un $54 \%(56 / 104)$, Bosco et al. ${ }^{15}$ manifestaron un 33,33\% $(10 / 30)$ y Okunseri et al. ${ }^{16}$ reportaron un $20,6 \%(21 / 102)$.

En este estudio se establecieron las categorías clínicas, hallándose en la categoría clínica $\mathrm{C}$ (signos y síntomas severos) la presencia de patología oral (herpes simple). El hallazgo coincide con lo reportado por Benito y et al., ${ }^{20}$ quienes señalaron que la mayoría de lesiones orales identificadas en su investigación se encontraban en la misma categoría clínica, 40,62\% (13/32). Sin embargo, Miziara et al. ${ }^{18}$ manifestaron que la mayor cantidad de lesiones orales en su estudio se encontraban en la categoría clínica A, $41 \%$ (188/459).

La patología encontrada en este estudio se localiza en un paciente con inmunosupresión severa. De igual manera Bosco et $a{ }^{15}$ reportaron que las manifestaciones orofaciales son más frecuentes en niños con inmunosupresión moderada y severa, Benito et al. ${ }^{20}$ mencionaron que las patologías bucales están relacionadas con una mayor depresión del sistema inmunitario, Okunseri et al. ${ }^{16}$ reportaron además que los niños con bajo porcentaje de CD4 tuvieron más lesiones orales.

En la presente investigación se observó que el $90 \%$ (18/20) de pacientes presentaba un tiempo de tratamiento mayor de un año, presentándose un solo caso de herpes simple. Por lo cual el tiempo de tratamiento es crucial para la reconstitución del sistema inmunitario, coincidiendo con Okunseri et al. ${ }^{16}$, Ferreira et al. ${ }^{19}$, Benito et al. ${ }^{20}$ y Miziara et al. ${ }^{18}$

Okunseri et al. ${ }^{16}$ manifestaron que los niños que reciben TARGA constantemente desde su nacimiento tienen una baja prevalencia de lesiones orales. Ferreira et al. ${ }^{19}$ informaron que el tiempo y el uso del tratamiento antirretroviral puede influir en la salud oral y sistémica del paciente. Benito et al. ${ }^{20}$ menciona- ron que el tiempo de uso del TARGA es fundamental y Miziara et al. ${ }^{18}$ reportaron que el tiempo del uso del TARGA podría estar asociado a una menor prevalencia de lesiones orales y un mayor recuento de linfocitos CD4.

La elección del tipo de tratamiento antirretroviral adecuado es muy importante para una mejora del estado clínico e inmunológico, compartiendo la opinión de Flanagan et al..$^{21}$, Gaitán et al., ${ }^{14}$ Ferreira et al. ${ }^{19}$ y Miziara et al. ${ }^{18}$ En relación con el tipo de esquema en el presente estudio, los pacientes pediátricos infectados por $\mathrm{VIH}$ reciben TARGA luego de ser evaluados según criterios establecidos, sin embargo algunos pacientes, debido a diversos factores, no responden satisfactoriamente al esquema dado, es entonces que se debe establecer un esquema de rescate suministrando el TARGA conveniente a cada situación.

\section{Conclusiones}

Se atribuye la presencia mínima de patología oral asociada al VIH al tratamiento antirretroviral de gran actividad que favorece la reconstitución del sistema inmunitario y mejoría en la condición clínica oral y sistémica.

Las lesiones cariosas y la gingivitis fueron relacionadas a la deficiencia de higiene oral.

\section{Referencias bibliográficas}

1. Unaids.org. ONUSIDA [sede Web]. Ginebra:Unaids.org; 2015- [actualizada el 14 de julio de 2015; acceso 10 de agosto de 2015]. Disponible en: http:// www.unaids.org/es

2. Holguín A, Soriano V. ¿Cuántos VIH existen? Med Clin (Barc). 1998; 110(17): 657-61.

3. Holguín A. Importancia de los subtipos genéticos del VIH en el mundo e implicaciones clínicas. Agenda de Acción [revista de Internet] 2006 marzo. [acceso 07 de junio de 2010]; I. Disponible en: http://www.fundacionfit. org/PDF/Agenda\%20de\%20Accion\%20\%20n_1_2006/TRATAMIENTO.pdf

4. Gonzáles N, Díaz J, Pérez A. La transmisión materno infantil VIH/ sida en Cuba. Rev Cubana Med Trop. 2000; 52(3): 220-4.

5. Gatell J, Clotect B, Podzamezer D, Miró J, Mallolas J. Guía práctica del SIDA, clínica, diagnóstico y 
tratamiento. $5^{\text {a }}$ ed. Barcelona: Ed. MASSON S.A; 1998.

6. Expósito J, Vallejos E, Martos E. Manifestaciones orales de la infección por VIH en la infancia: Artículo de revisión. Med Oral Patol Oral Cir Bucal. 2004; 9: 410-20.

7. Gómez J. Infección por VIH en el recién nacido y lactante una situación evitable. BSCP Can Ped. 2000; 24(3): 181-190.

8. Pachón J, Pujol E, Rivero A. Editores. La infección por VIH: Guía práctica. $2^{a}$ ed. Sevilla: Sociedad Andaluza de Enfermedades Infecciosas; 2003.

9. Ministerio de Salud del Perú. Atención integral y tratamiento antirretroviral de los nińos, niñas y adolescentes infectados por el virus de la inmunodeficiencia humana 2009. Lima: Ministerio de Salud del Perú, Dirección de Servicios de Salud; 2009.

10. Francisco J, Ramos G, Catherine F, Catapano P, Murray P, Milens A, et al. Classification, diagnostic, criteria and treatment recommendations for orofacial manifestation in HIV-infected pediatric patiens. J ClinPediatr Dent. 1999; 23(2): 85-96.

11. Dos Santos R, Tenorio T, Beder C, Carneiro J, Pomarico I, Fernanda G. Oral manifestations in human immunodeficiency virus infected children in higly active antiretroviral therapy era. J Oral Pathol Med. 2009; 38: 613-622.
12. Bascones A, Serrano C, Campo J. Manifestaciones de la infección por el virus de la inmunodeficiencia humana en la cavidad bucal. Med Clin (Barc). 2003; 120(11): 426-34.

13. Ramos F. Dental consideration for the paediatric AIDS/HIV patient. Oral Dis. 2002; 8 Supl.2: 49-54.

14. Gaitán C, Borgues A, Martínez A, Fragoso R, Cuairán V, Casta M, et al. Lesiones orales en niños $\mathrm{VIH}+1$ SIDA bajo dos diferentes tipos de terapias. Bol. Med. Hosp. Infant. Mex. 2001; 58(1): 12-20.

15. Bosco V, Birman E. Oral manifestations in children with AIDS and in controls. Pesqui Odontol Bras. 2002; 16(1): 07-11.

16. Okunseri C, Badner V, Wiznia A, Rosenberg M. Prevalence of oral lesions and percent CD4+ T- lymphocytes in HIV - infected children on antiretroviral therapy. Patient Care STDS. 2003; 17(1): 5-11.

17. Pongsiriwet S, Iamaroon A, Kanjanavanit S, Pattanaporn K, Krisanaprakornkit S. Oral lesions and dental caries status in perinatally HIV-infected children in Northern Thailand. Int J Paediatr 2003; 13(3): 180-5.

18. Miziara I, Filho B, Weber R. Oral lesions in Brazilian HIV-infected children undergoing HAART. Int J Pediatr Otorhinolaryngol. 2006; 70(6): 1089-96.

19. Ferreira L, Barbosa G, Ribeiro I, Pinheiro M. Pediatric HIV-related oral manifestation- a five-year re- trospective study Braz. Oral Res. 2004; 18(1): 06-11.

20. Benito M, Rojas T, GarcíaR, Villalobos H, RiveraN, Bernardoni C, et al. Relación entre las manifestaciones bucales asociadas al VIHSIDA pediátrico y las categorías clínicas inmunológicas. Ciencia Odontológica. 2005; 2(1): 34-46.

21. Flanagan M, Barasch A, Koenigsberg S, Fine D, Houpt M. Prevalence of oral soft tissue lesions in HIV-infected minority children treated with highly active antiretroviral therapies. AAPD. 2000; 22(4): 287-291.

22. Khongkunthian P, Grote M. Isaratanan W, Pyaworawong S, Reichart P. Oral manifestations in $45 \mathrm{HIV}$ - positive children from Norther Thailand. J Oral Pathol Med. 2001; 30(9):549-552.

23. Chen J, Flaitz C, Wullbrandt B, Sexton J. Association of dental health parameters with oral lesions prevalence in human immunodeficency virus - infected Romanian children. Pediatr. Dent. 2003; 25(5): $479-484$

24. Hanza O, Matee M, Simon E, Kikwlu E, Moshi M, Mugusi F, et al. Oral Manifestation of infection in children and adults receiving highly active antiretroviral therapy (HAART) in Dar es Salaam, Tanzania. BMC Oral Health. 2006; 6-12.

25. Leggott J. Oral manifestations of HIV infection in children. Oral Surg Oral Med Oral Pathol. 1992; 73: 187-92. 